Received: 30.08 .2021

Revised: 30.09 .2021

Accepted: 29.10 .2021

DOI: $10.17804 / 2410-9908.2021 .5 .006-014$

\title{
FEATURES OF MAGNETOSTRICTION IN CARBON STEELS
}

\author{
V. F. Novikov ${ }^{\text {a) }}$, K. R. Muratov ${ }^{\text {b) }}$, S. M. Kulak ${ }^{\text {c)* }}$, \\ A. S. Parakhin ${ }^{\text {d) }}$, and R. A. Sokolov ${ }^{\text {e) }}$ \\ Tyumen Industrial University, \\ 38 Volodarskogo St., Tyumen, 625000, Russian Federation \\ a) iD http://orcid.org/0000-0002-1987-351X vitaly.nowikov2017@yandex.ru; \\ b) iD http://orcid.org/0000-0002-8079-2022 muratows@ mail.ru; \\ c) iD http://orcid.org/0000-0002-5970-8893 ksm-rabochi@rambler.ru; \\ d) $\otimes$ paskgn@mail.ru; \\ e) iD http://orcid.org/0000-0001-5867-8170 $ه$ falcon.rs@mail.ru \\ *Corresponding author. E-mail: ksm-rabochi@rambler.ru \\ Address for correspondence: ul. Melnikaite, 70, room 317, Tyumen, 625039, Russian Federation \\ Tel.: 89829247897
}

Magnetostriction of the tempered 60G steel in the magnetic field of a solenoid is studied. A technique is proposed for dividing magnetostriction into components, namely those induced by the displacement of interdomain boundaries and those caused by the mechanism of rotation of spin magnetic moments. The dependences of the magnetostriction components on the tempering temperature of the hardened steel are constructed. This contributes to an increase in the effectiveness (accuracy) of evaluating elastic stresses in a steel structure by magnetoelastic methods. The essence of the technique lies in the application of the Langevin function to describing the experimental magnetostriction curves of the tempered 60G steel.

Keywords: magnetostriction, displacement of domain boundaries, magnetic rotation of magnetic moments, ferromagnetic, elastic stresses.

\section{References}

1. Akulov N.S. Ferromagnetizm [Ferromagnetism]. Moscow-Leningrad, Gostekhteoretizdat Publ., 1939, 188 p. (In Russian).

2. Vonsovsky S.V., Shur Ya.S. Ferromagnetizm [Ferromagnetism]. Moscow-Leningrad, OGIZ-Gostekhizdat Publ., 1948, 648 p. (In Russian).

3. Bozort R. Ferromagnetizm [Ferromagnetism]. Moscow, Izd-vo inostr. lit., 1956, 784 p. (In Russian).

4. Novikov V.F., Bakharev M.S. Magnitnaya diagnostika mekhanicheskih napryazhenii [Magnetic Diagnostics of Mechanical Stresses]. Tyumen, Izdatelstvo Vector BUK, 2001, 220 p. (In Russian).

5. Muratov K.R., Novikov V.F., Neradovskii D.F., Kazakov R.K. Magnetoelastic demagnetization of steel under cyclic loading. The Physics of Metals and Metallography, 2018, vol. 119 (1), pp. 18-25. DOI: 10.1134/S0031918X1801012X.

6. Novikov V.F., Yatsenko T.A., Bakharev M.S. On the nature of the piezomagnetic effect of the remanently magnetized state of a magnet. Izvestiya Vuzov, Neft i Gaz,1998, No. 4, pp. 96-102 (In Russian). 
7. Deordiev G.I., Biktashev T.Kh. Magnetostriction method of measuring stresses in components of metallic structures. Soviet Journal of Nondestructive Testing-USSR, 1977, no. 3, pp.83-91.

8. Gorkunov E.S., Subachev Yu.V., Povolotskaya A.M., Zadvorkin S.M. The influence of an elastic uniaxial deformation of a medium-carbon steel on its magnetostriction in the longitudinal and transverse directions. Russian Journal of Nondestructive Testing, 2013, No. 10, pp. 40-52. DOI: $10.1134 / \mathrm{S} 1061830913100057$.

9. Gorkunov E.S., Povolotskaya A.M., Soloviev K.E., Zadvorkin S.M. The influence of the magnetoelastic effect on the hysteretic properties of medium-carbon steel during uniaxial loading. Russian Journal of Nondestructive Testing, 2010, 46 (9), pp. 638-644. DOI: 10.1134/S1061830910090032.

10. Novikov V.F., Kostryukova N.K., Nassonov V.V. Fedorov B.V., Rybnikova O.I. Change in the magnetostriction of certain steels during the initial stages of plastic deformation. Russian Journal of Nondestructive Testing, 1996, vol. 32, iss. 5, pp. 418-422.

11. Novikov V.F., Tikhonov V.F. On Studying Fatigue Changes in Metal upon Bending Vibrations of Blades by Magnetic and Magnetoelastic Methods. Probl. Prochn., 1981, no. 5, pp. 13-17. (In Russian).

12. Gorkunov E.S., Povolotskaya A.M., Zadvorkin S.M., Putilova E.A., Mushnikov A.N., Bazulin E.G., Vopilkin A.Kh. Some Features in the Behavior of Magnetic and Acoustic Characteristics of HotRolled 08G2B Steel under Cyclic Loading. Russian Journal of Nondestructive Testing, vol, 55, iss. 11, pp. 827-836. DOI: 10.1134/S1061830919110044.

13. Tagaki M. On a Statistical Domain Theory of Ferromagnetic Crystals. Part II. Sci. Rep. Tohoka Imp. Univ., 1939, vol. 28, pp. 85-127.

14. Mishin D.D., Novikov V.F., Kurdyumov V.G. The influence of the dislocation structure on the coercive force of siliceous iron Fizika metallov $i$ metallovedenie, 1967, vol. 24, iss. 1, pp. 175-177. (In Russian).

15. Tamm I.E. Osnovy teorii elektrichestva [Fundamentals of the theory of electricity: Manual for Universities, $11^{\text {th }}$ ed., rev. and add.]. Moscow, Fizmatlit Publ., 2003, 616 p. (In Russian).

16. Kalitkin N.N. Chislennye metody [Numerical Methods]. Moscow, Nauka Publ., 1978, 508 p. (In Russian).

17. Parakhin A.S. EVM v laboratornom praktikume [Computer in Laboratory Practice: Tutorial]. Kurgan, Izd-vo Kurg. Gos. Un-ta, 2000, 109 p. (In Russian).

18. Ulyanov A.I., Elsukov E.P., Chulkina A.A., Zagainov A.V., Arsentyeva N.B., Konygin G.N., Novikov V.F., Isakov V.V. The role of cementite in the formation of magnetic hysteresis properties of plastically deformed high-carbon steels: I. Magnetic properties and structural state of cementite. Russian Journal of Nondestructive Testing, 2006, vol. 42, No. 7, pp. 452-459. DOI: $10.1134 / \mathrm{S} 1061830906070047$. 
Подана в журнал: 30.08.2021

УДК 537.622.4:537.634.2

DOI: $10.17804 / 2410-9908.2021 .5 .006-014$

\title{
ОСОБЕННОСТИ МАГНИТОСТРИКЦИИ В УГЛЕРОДИСТЫХ СТАЛЯХ
}

\author{
В. Ф. Новиков ${ }^{\text {a) }}$, К. Р. Муратов ${ }^{\text {()) }}$, С. М. Кулак ${ }^{\text {в)* }}$, \\ А. С. Парахин ${ }^{\text {г) }}$, Р. А. Соколов ${ }^{\text {д) }}$ \\ Федеральное государственное бюджетное образовательное учреждение высшего образования \\ Тюменский индустриальный университет, \\ д. 38, ул. Володарского, г. Тюмень, Российская Федераџия \\ a) iD http://orcid.org/0000-0002-1987-351X vitaly.nowikov2017@yandex.ru; \\ б) (iD http://orcid.org/0000-0002-8079-2022 muratows@ mail.ru; \\ в) iD http://orcid.org/0000-0002-5970-8893 ksm-rabochi@rambler.ru; \\ г) $\otimes$ paskgn@mail.ru; \\ д) (iD http://orcid.org/0000-0001-5867-8170 $\otimes$ falcon.rs@mail.ru \\ *Ответственный автор. Эл. почта: ksm-rabochi@rambler.ru \\ Адрес для переписки: ул. Мельникайте 70, ауд. 317, г. Тюмень, 625039, Российская федерация \\ Тел.:8-982-924-78-97
}

Проведены исследования магнитострикции стали 60Г в магнитном поле соленоида после ее отпуска. Предложена методика разделения магнитострикции на составляющие, вызванные смещением междоменных границ и механизмом вращения спиновых магнитных моментов. Построены зависимости составляющих магнитострикции от температуры отпуска закаленной стали. Это способствует повышению эффективности (точности) оценки упругих напряжений в стальной конструкции магнитоупругими методами. Суть методики заключена в применении функции Ланжевена для описания экспериментальных кривых магнитострикции исследуемой стали 60 п прошедшей отпуск.

Ключевые слова: магнитострикция, смещение доменных границ, магнитное вращение магнитных моментов, ферромагнетик, упругие напряжения.

\section{1. Введение}

Магнитострикция является одним из важных параметров, входящих в выражение для магнитоупругой энергии ферромагнетиков [1-3]. Она сказывается на кривых намагничивания [2-3], параметрах петли гистерезиса (коэрцитивной силе, остаточной намагниченности и др.) и их изменениях при механическом воздействии [1-5], лежит в основе магнитоупругого размагничивания и пьезомагнитного эффекта остаточно намагниченного состояния [4-6]. Полевая зависимость магнитострикции $\lambda(\mathrm{H})$, зависит от химического состава и вида термообработки [2-4], упругой [6-7], пластической [3, 8], усталостной деформации [8-10] и чувствительна к различным текстурам (кристаллической текстуре, ориентированным напряжениям, термомагнитной обработке) [2-4].

Типичный вид зависимости магнитострикции железа и низкоуглеродистых слаболегированных сталей представлен в виде кривых $\lambda(\mathrm{H}, \sigma)$ (рис. 1). Экспериментальные результаты, начиная с точки перегиба, апроксимировались функцией:

$$
\lambda=\mathrm{C}_{1} L\left(\alpha_{1} H\right)+\mathrm{C}_{2},
$$

где $L(x)=\operatorname{cth}(x)-\frac{1}{x}$ 


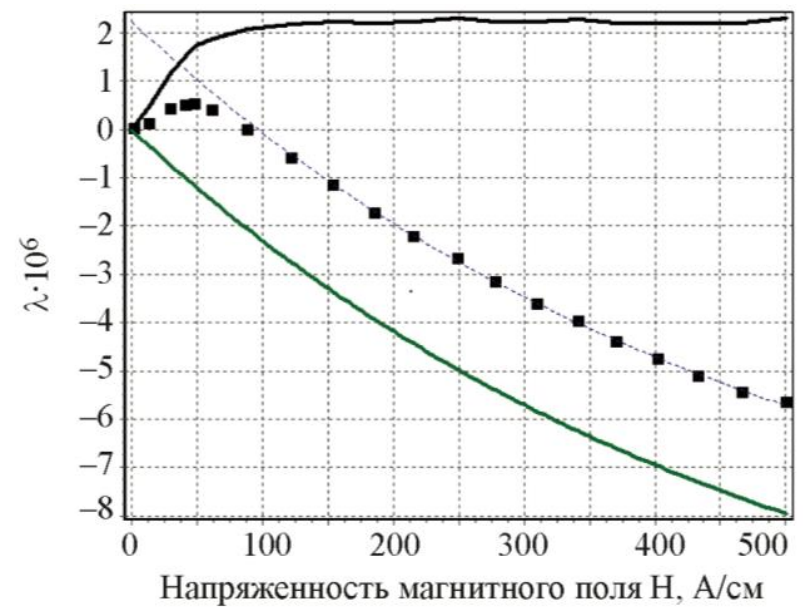

$a$

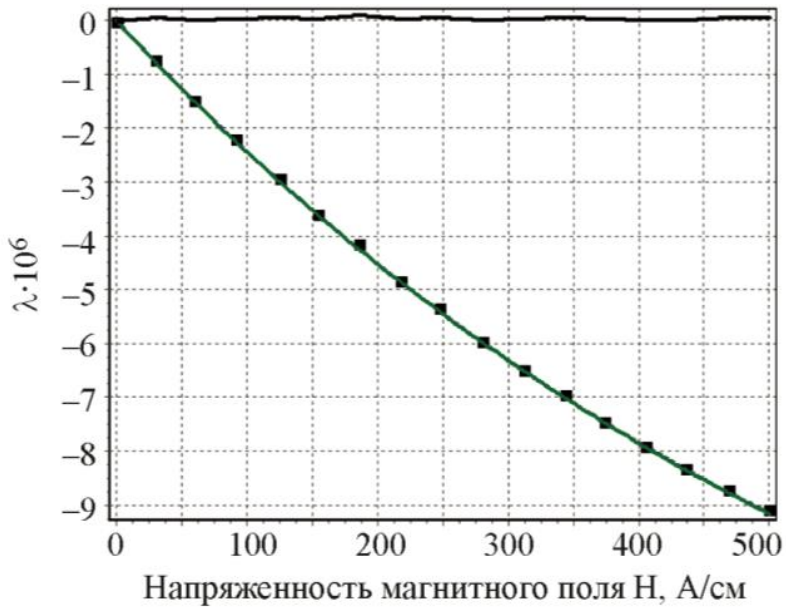

$\sigma$

Рис. 1. Полевая зависимость магнитострикции $\lambda$ стали 45 (точки) [8]: $a-$ в ненагруженном состоянии; $\sigma$ - под действием растягивающего напряжения 209 МПа. Сплошная линия -

кривая аппроксимации (кривая Ланжевена) с параметрами: $a-\mathrm{C}_{1}=12,3395 \cdot 10^{-6}$; $\mathrm{C}_{2}=-10,1060 \cdot 10^{-6} ; \sigma_{1}=-0,00206$; отклонение $0,011 \cdot 10^{-6}$; экстраполяция $2,23 \cdot 10^{-6}$; $\sigma-\mathrm{C}_{1}=17,0631 \cdot 10^{-6} ; \mathrm{C}_{2}=-17,0269 \cdot 10^{-6} ; \alpha_{1}=-0,001540 ;$ отклонение $0,007 \cdot 10^{-6}$; экстраполяция $0,0362 \cdot 10^{-6}$

С ростом поля магнитострикция ненагруженного железа или стали достигает положительного максимума, убывает, проходит через ноль, становится отрицательной и в больших полях достигает насыщения (рис. $1 a$ и 1 б). При приложении растягивающих напряжений положительная составляющая магнитострикции уменьшается по экспоненте [13] и при напряжениях порядка 50-300 МПа полевая зависимость магнитострикции вырождается в кривую обусловленную процессами вращения. В теории ферромагнетизма это объясняется конкуренцией двух механизмов намагничивания: механизма смещения междоменных границ и механизма вращения спиновых магнитных моментов (в дальнейшем механизмом смещения и вращения). Магнитострикция насыщения $\lambda \mathrm{s}$ изотропного поликристаллического железа определяется выражением[1-2].

$$
\lambda_{\mathrm{s}}=\left(2 / 5 \cdot \lambda_{100}+3 / 5 \cdot \lambda_{111}\right)
$$

где $\lambda_{100}$ и $\lambda_{111} \ldots$. константы, определяющие величину магнитострикции. Так как константа магнитострикции железа $\lambda_{111}$ имеет отрицательное значение, то $\lambda_{\mathrm{s}}$ является разностью двух независимых функций магнитострикций.

Раздельный анализ кривой магнитострикции проводился главным образом на поликристаллических материалах монокристаллах железа, никеля, кремнистого железа, железакобальтом и др. $[2,3,10,13]$. Однако в основном используется экспериментальная (интегральная) кривая магнитострикции и изучаются закономерности ее изменения в зависимости от напряжений, от структуры, марки стали и вида отжига [2, 3]. В работе [4] была сделана попытка разделить экспериментальную кривую магнитострикции на составляющие графическим способом и использовать их для учета чувствительности коэрцитивной силы к упругим напряжениям. Разность составляющих скрывает их значение, поэтому их разделение может быть полезно для оценки абсолютной величины составляющих, выяснения их роли в магнитоупругих явлениях. Зависимость составляющей магнитострикции сталей, обусловленной процессами вращения слабо изучена. В частности, не определена ее чувствительность к упругим напряжениям и пластической деформации. 


\section{2. Постановка задачи и методы решения}

Цель работы - поиск более объективного способа разделения кривой полевой зависимости магнитострикции на составляющие: положительной, обусловленной процессами смещения, и отрицательной, вызванной вращением векторов намагничения, оценить на примере стали 60Г влияние термической обработки на изменения этих составляющих. В качестве материалов для исследования были выбраны сталь 60Г и сталь 45 [9]. Магнитострикция измерялась в магнитном поле соленоида на стандартных образцах, длиной 10 мм и диаметром 8 мм, мостовым методом с помощью наклеиваемых тензодатчиков. Калибровка осуществлялась на никелевом электролитическом эталоне. Коэрцитивная сила определялась в магнитометрической установке. Для анализа использовались данные полученные в работе по изучению влияния растягивающих напряжений на кривую магнитострикции [9].

В работе [4] предложено выразить экспериментально снимаемую зависимость магнитострикции от напряженности поля $\lambda(\mathrm{H})$ сталей [4] в виде суммы двух монотонных функций.

$$
\lambda_{\text {эксп }}(\mathrm{H})=\lambda_{\text {см}}(\mathrm{H})(\text { кривая } 1)+\lambda_{\text {вр }}(\mathrm{H})
$$

где $\lambda_{\text {см }}(\mathrm{H})$ (кривая 1) и $\lambda_{\text {вр }}(\mathrm{H})$ пропорциональны константам $\lambda_{100}$ и $\lambda_{111}$ соответственно. Первая функция обусловлена перестройкой доменной структуры (поперечной в продольную) путем смещения междоменных 90-градусных границ, вторая - процессом поворота (вращения) векторов намагничивания от направления легких осей до направления магнитного поля. На кристаллах железа и кремнистого железа $[2,13,14]$ для направления [100] показано, что кривая полевой зависимости магнитострикции похожа на тангенс гиперболи-

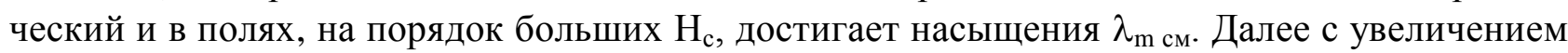
поля $\lambda_{\mathrm{m}}$ см практически не изменяется. В поликристаллическом железе максимальное отклонение наилегчайшей оси от направления поля не превышает 55 градусов, поэтому необходимое для насыщения поле, как минимум для такого же кристаллита, должно быть не

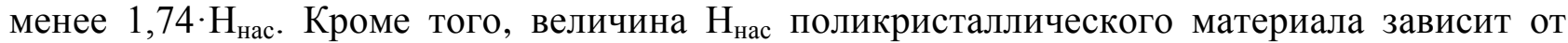
структуры и внутреннего размагничивающего фактора и формы образца. Мы исходим из того, что в полях на порядок больших, чем коэрцитивная сила (порядка 200-4000 А/м), магнитострикция смещения достигает насыщения и перестает вносить изменения в кривую экспериментальной магнитострикции и последняя будет представлять собой кривую отрицательной составляющей магнитострикции сдвинутой вверх на величину $\lambda_{\mathrm{m}}$ см. $\mathrm{B}$ работах $[2,7,9]$ показано, что при растяжении железа положительная составляющая магнитострикции $\lambda_{\mathrm{m} \text { см }}$ смещения уменьшается по экспоненте [13], при напряжениях порядка 50-300 МПа вырождается в кривую, пример которой показан на рис. 1 б. Кривые ниспадающей магнитострикции опускаются вниз почти параллельно друг другу с ростом растягивающих напряжений. При сжимающих напряжениях вся кривая поднимается вверх. Рост или уменьшение положительной магнитострикции сдвигает составляющую магнитострикции вращения вниз или вверх.

В предлагаемой работе был проведен поиск аппрокимирующих функций. Рассмотрена функция Ланжевена (3) для ферромагнетиков (магнитных диполей) которая хорошо согласуется с экспериментом [15]. Обработка экспериментальных результатов проводилась по методике, описанной в работах $[16,17]$. Пример аппроксимации для стали 60Г, отпущенной при $700{ }^{\circ} \mathrm{C}$, представлен на рис. 2. Видно хорошее согласие функции Ланжевена с экспериментальными результатами (квадратики). 


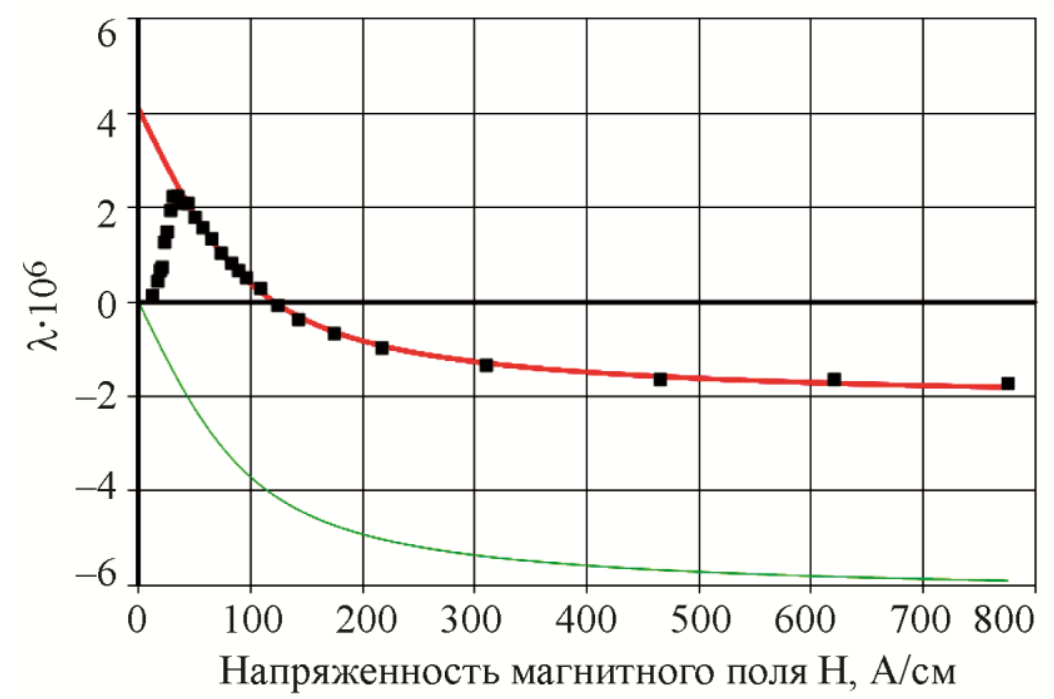

Рис. 2. Сталь 60Г. Отпуск $700{ }^{\circ} \mathrm{C}$. Параметры: $\mathrm{C}_{1}=-6,2389 \cdot 10^{-6} ; \mathrm{C}_{2}=4,0963 \cdot 10^{-6}$; $\alpha_{1}=-0,02355$; отклонение $0,013 \cdot 10^{-6}$

В предлагаемом анализе вторая половина графика (диапазон больших полей) является кривой, поднятой вверх на величину положительной составляющей магнитострикции.

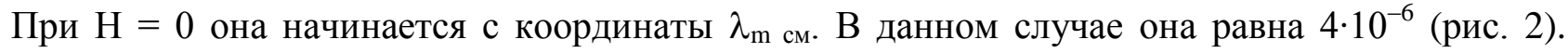
Сдвинутая вниз на $\lambda_{\mathrm{m}}$ см она будет представлять собой начинающую отсчет от нуля отрицательную составляющую.

По результатам разложения экспериментальных кривых для максимальных полей были построены четыре графика (рис. 3): зависимость положительных значений магнитострикции в максимуме (пиковых) $\lambda_{\text {п }}$, отрицательных значений магнитострикции в больших полях $\lambda_{\mathrm{s}}$, расчетного значения $\lambda_{\mathrm{m}}$ см и отрицательной составляющей магнитострикции, полученной экспериментально в наибольшем поле $\lambda_{\mathrm{m}}$ вр от температуры отпуска закаленной стали $60 Г$.

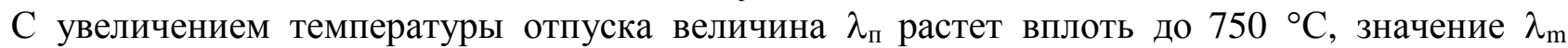
колеблется для диапазона $400-750{ }^{\circ} \mathrm{C}$ вблизи значений $2 \cdot 10^{-6}$. Расчетное значение составля-

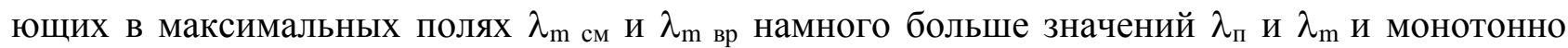
увеличивается с ростом температуры отпуска (рис. 3).

Перлитно-ферритная сталь после высокотемпературного отпуска состоит из цементита и феррита и увеличивается с ростом температуры отпуска. В чистом виде компонента

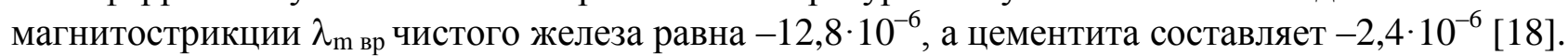
С ростом температуры отпуска увеличивается количество ферритной фазы. Об этом говорит уменьшение коэрцитивной силы (рис. 4). Однако магнитострикция смеси не сможет достигнуть магнитострикции ферритной фазы, так как фазы цементита и феррита сопряжены друг с другом, а их величины существенно отличаются, поэтому магнитострикция ферритного объема будет испытывать сжимающие напряжения, растягивая сопряженную с ней фазу цементита.

На рис. 5 приведены графики полевых зависимостей магнитострикции длинных образцов из сталей 15 ХСНД и 25 ХСНД в состоянии поставки. Существенное различие кривых обусловлено не только их химическим составом, но и их кристаллографической и магнитной текстурой формируемой конечной термообработкой. Проведенное разложение на составляющие для максимальных полей (таблица)показывает, что основные составляющие близких по составу сталей различаются не так сильно, как их экспериментально установленные (разностные) параметры. 


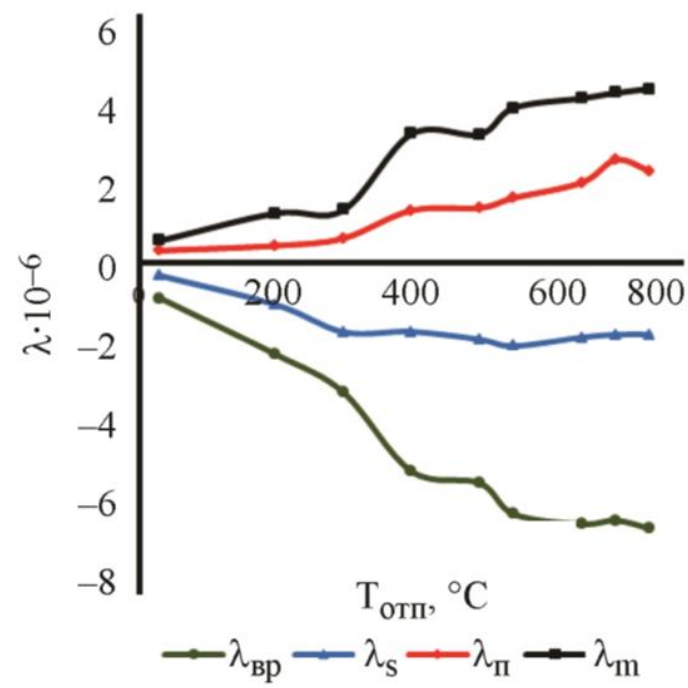

Рис. 3. Зависимость магнитострикции в максимуме $\left(\lambda_{\text {п }}\right)$ и в максимальном поле $\left(\lambda_{\mathrm{s}}\right)$, составляющих в максимуме смещения $\left(\lambda_{\mathrm{m}}\right)$ и вращения $\left(\lambda_{\text {вр }}\right)$ от температуры отпуска стали $60 \Gamma$

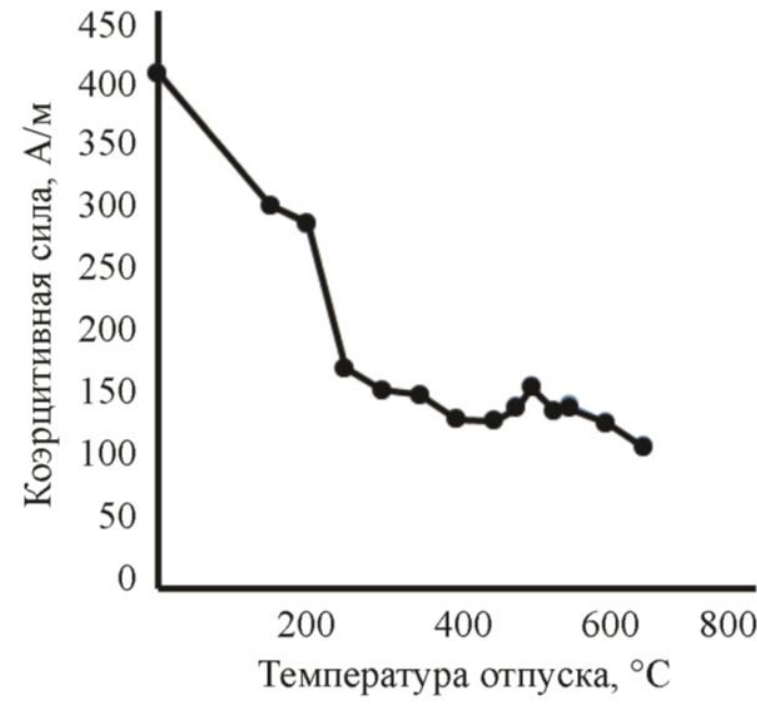

Рис. 4. Зависимость коэрцитивной силы закаленной стали 60Г от температуры отпуска

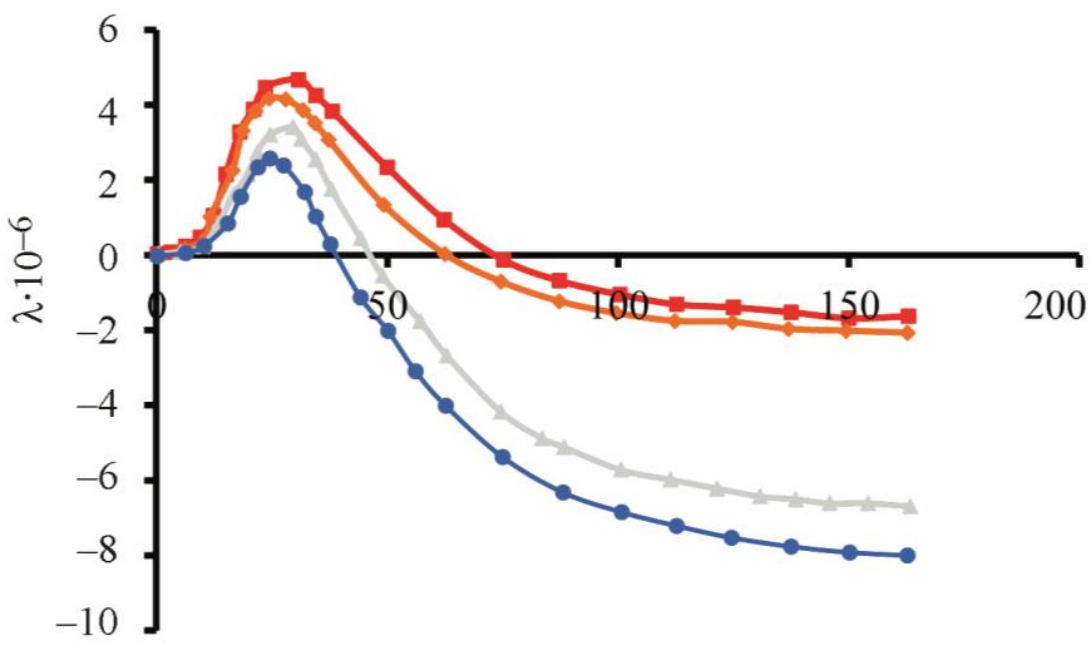

$\mathrm{H}, \kappa \mathrm{A} / \mathrm{M}$

Рис. 5. Полевая зависимость магнитострикции для трубных сталей: 1 - 15ХСНД; 2 - 15ХСНД; 3 - 25ХСНД; 5 - 25ХСНД

Таблица - Положительная $\lambda_{\mathrm{m} \text { см }}$ и отрицательная $\lambda_{\text {вр }}$ составляющие магнитострикции $\lambda_{\mathrm{s}}$

\begin{tabular}{|l|c|c|c|}
\hline Марка стали & $\lambda_{\mathrm{s}} \cdot 10^{-6}$ & $\lambda_{\mathrm{m} \mathrm{cм}} \cdot 10^{-6}$ & $\lambda_{\text {вр }} \cdot 10^{-6}$ \\
\hline 15 ХСНД & 1,45 & 8,3 & 9,8 \\
\hline 15 ХСНД & 2,0 & 8,6 & 10,6 \\
\hline 25 ХСНД & 6,7 & 9,0 & 15,7 \\
\hline 25 ХСНД & 8,0 & 9,1 & 17,1 \\
\hline
\end{tabular}




$$
\lambda_{100}=\left(\frac{5}{2} \cdot \lambda_{m}\right), \lambda_{111}=-\left(5 / 3 \cdot \lambda_{\text {вр }}\right)
$$

Располагая результатами разложения, можно оценивать в соответствии (1) константы магнитострикции низкоуглеродистых сталей в изотропном поликристаллическом состоянии.

\section{3. Заключение}

Предложен и реализован способ определения составляющих магнитострикции низкоуглеродистых сталей в изотропном состоянии.

Выделение компонент магнитострикции стали позволит более точно определять магнитоупругую чувствительность коэрцитивной силы и остаточной намагниченности сталей к упругим напряжениям.

\section{Литература}

1. Акулов Н. С. Ферромагнетизм. - Москва ; Ленинград : Гостехтеоретиздат, 1939. - 188 с.

2. Вонсовский С. В., Шур Я. С. Ферромагнетизм. - Москва-Ленинград : ОГИЗГостехиздат, 1948. - 648 с.

3. Бозорт Ричард М. Ферромагнетизм / пер. с англ. под ред. Е. И. Кондорского и Б. Г. Лившица. - Москва : Изд-во иностр. лит., 1956. - 784 с.

4. Новиков В. Ф., Бахарев М. С. Магнитная диагностика механических напряжений. Тюмень : Издательство Вектор БУК, 2001. - 220 с.

5. Magnetoelastic demagnetization of steel under cyclic loading / K. R. Muratov, V. F. Novikov, D. F. Neradovskii, R. K. Kazakov // The Physics of Metals and Metallography. - 2018. Vol. 119 (1). - P. 18-25. - DOI: 10.1134/S0031918X1801012X.

6. Новиков В. Ф., Яценко Т. А., Бахарев М. С. К природе пьезомагнитного эффекта остаточно намагниченного состояния магнетика // Изв. вуз. Нефть и газ. - 1998. - № 4. C. 96-102.

7. Deordiev G. I., Biktashev T. Kh. Magnetostriction method of measuring stresses in components of metallic structures // Soviet Journal of Nondestructive Testing-USSR. - 1977. - No. 3. P. 83-91.

8. The influence of an elastic uniaxial deformation of a medium-carbon steel on its magnetostriction in the longitudinal and transverse directions / E. S. Gorkunov, Yu. V. Subachev, A. M. Povolotskaya, S. M. Zadvorkin // Russian Journal of Nondestructive Testing. - 2013. No. 10. - P. 40-52. - DOI: 10.1134/S1061830913100057.

9. The influence of the magnetoelastic effect on the hysteretic properties of medium-carbon steel during uniaxial loading / E. S. Gorkunov, A. M. Povolotskaya, K. E. Soloviev, S. M. Zadvorkin // Russian Journal of Nondestructive Testing. - 2010. - Vol. 46 (9). - P. 638-644. DOI: $10.1134 / \mathrm{S} 1061830910090032$.

10. Change in the magnetostriction of certain steels during the initial stages of plastic deformation / V. F. Novikov, N. K. Kostryukova, V. V. Nassonov, B. V. Fedorov, O. I. Rybnikova // Russian Journal of Nondestructive Testing. - 1996. - Vol. 32, iss. 5. - P. 418-422.

11. Новиков В. Ф., Тихонов В. Ф. К изучению усталостных изменений в металле при изгибных колебаниях лопаток турбин магнитным и магнитоупругим методами // Проблемы прочности. - 1981.- № 5. - С. 13-17.

12. Some Features in the Behavior of Magnetic and Acoustic Characteristics of Hot-Rolled 08G2B Steel under Cyclic Loading / E. S. Gorkunov, A. M. Povolotskaya, S. M. Zadvorkin, 
E. A. Putilova, A. N. Mushnikov, E. G. Bazulin, A. Kh. Vopilkin // Russian Journal of Nondestructive Testing. - Vol, 55, iss. 11. - P. 827-836. - DOI: 10.1134/S1061830919110044.

13. Takagi M. On a statistical Domain theory of Ferromagnetic cristals. Part I // Sci. Rep. Tohoku Imp. Univ. - 1939. - Vol. 28. - P. 85-127.

14. Мишин Д. Д., Новиков В. Ф., Курдюмов В. Г. Влияние дислокационной структуры на коэрцитивную силу кремнистого железа // ФММ. - 1967.- Т. 24, вып. 1. - С. 175-177.

15. Тамм И. Е. Основы теории электричества: учебное пособие для вузов. - 11-е изд., испр. и доп. - Москва : ФИЗМАТЛИТ, 2003. - 616 с.

16. Калиткин Н. Н. Численные методы. - Москва : Наука, 1978. - 508 с.

17. Парахин А. С. ЭВМ в лабораторном практикуме : учебное пособие. - Курган : Изд-во Кург. гос. ун-та, Курган, 2000. - 109 с.

18. The role of cementite in the formation of magnetic hysteresis properties of plastically deformed high-carbon steels: I. Magnetic properties and structural state of cementite / A. I. Ulyanov, E. P. Elsukov, A. A. Chulkina, A. V. Zagainov, N. B. Arsentyeva, G. N. Konygin, V. F. Novikov, V. V. Isakov // Russian Journal of Nondestructive Testing. - 2006. - Vol. 42, No. 7. - P. 452-459. - DOI: 10.1134/S1061830906070047. 